\title{
LIMIT CYCLES AND INVARIANT CENTERS FOR AN EXTENDED KUKLES SYSTEM
}

\author{
ZHENHAI LIU AND IVÁN SZÁNTÓ
}

Received 08 June, 2016

\begin{abstract}
A class of polynomial systems of odd degree with limit cycles, invariant centers and invariant straight lines, is examined. The limit cycles can be obtain as a bifurcation of a non hyperbolic focus at the origin as Hopf bifurcations. We will also obtain the necessary and sufficient conditions for the critical point at the interior of bounded region to be a center.
\end{abstract}

2010 Mathematics Subject Classification: 92D25; 34C; 58F14; 58F21

Keywords: limit cycle, center, bifurcation

\section{INTRODUCTION}

Let us consider a real autonomous system of ordinary differential equations on the plane with polynomial nonlinearities.

$$
\left\{\begin{array}{l}
\dot{x}=P(x, y)=\sum_{i+j=0}^{n} a_{i j} x^{i} y^{j} \\
\dot{y}=Q(x, y)=\sum_{i+j=0}^{n} b_{i j} x^{i} y^{j}
\end{array}, \quad \text { with } a_{i j}, b_{i j} \in \mathbb{R}\right.
$$

Suppose that the origin of (1.1) is a critical point of center-focus type. We are concerned with two closely related questions, both of which are significant elements in work on Hilbert's 16th problem. The first is the number of limit cycles (that is, isolated periodic solutions) which bifurcate from a critical point and the second is the derivation of necessary and sufficient conditions for a critical point to be a center (that is, all orbits in the neighborhood of the critical point are closed). The limit cycles problem and the center problem is concentrated on specific classes of systems, those may be systems of a given degree (quadratic systems or a cubic systems, for example), or they may be of a particular form; for instance, a Kukles systems - that is, a systems of the form

$$
\dot{x}=-y, \quad \dot{y}=x+\lambda y+g(x, y) .
$$

Recall that systems of this type were studied, for the first time, by Isaak Solomonovich Kukles [4], who studied a linear center with cubic nonhomogeneous nonlinearities. A closely related problem is the derivation of conditions under which the system is 
integrable and other questions of interest relate to the coexistence of limit cycles and algebraic invariant curves.

Let $n=\max (\partial P, \partial Q)$, where the symbol $\partial$ denotes 'degree of'. A function $F$ is said to be invariant with respect to (1.1) if there is a polynomial $G$ called cofactor, with $\partial G<m$, such that $\dot{F}=F G$. Here $\dot{F}=F_{x} P+F_{y} Q$ is the rate of change of $F$ along orbits. It is well known that the existence of invariant curves has significant repercussions on the possible phase-portraits of the system.

Recently in [3], the authors considered a Kukles extended system of degree three, namely, a system of the form

$$
\left\{\begin{array}{l}
\dot{x}=P(x, y)=\lambda x+y+k x y \\
\dot{y}=Q(x, y)=-x+\lambda y+\sum_{i+j=2}^{3} a_{i j} x^{i} y^{j} \quad, \quad \text { with } k, a_{i j} \in \mathbb{R}
\end{array}\right.
$$

and the authors obtained some conditions for the existence of centre and limit cycles.

With the same idea, we consider an extended Kukles system of degree $2 n+5$, $n \geq 1$, with limit cycles, invariant parabolas and invariant straight lines, given by

$$
X_{\mu}:\left\{\begin{array}{l}
\dot{x}=-y\left(1-k^{2} x^{2}\right) \\
\dot{y}=\frac{1}{k^{2}}\left[2 x\left(1-k^{2} x^{2}\right)^{2}+\left(-1+k^{2} x^{2}-k^{2} y\right)\left(-1+k^{2} x^{2}+k^{2} y\right)\right. \\
\left.\quad\left(k^{2} x-2 x+k^{2} \lambda y+\sum_{i=1}^{n} a_{2 i+1} y^{2 i+1}\right)\right]
\end{array}\right.
$$

where $\mu=\left(k, \lambda, a_{3}, \ldots, a_{2 n+1}\right) \in \mathbb{R}^{n+2}, k \neq 0$. In this paper, for $n \geq 1$, we consider another class of extended Kukles system of degree $2 n+3$, with an invariant nondegenerate centers and two invariant straight line. For these kind of second-order differential systems, we show that for certain values of the parameters, the invariant centers and the invariant straight lines, can coexist with at least $n$ small amplitude limit cycles which are constructed by Hopf bifurcation. We conclude with some numerical simulations of the results.

\section{MAIN RESULTS}

Let us consider the extended Kukles of degree $2 n+3$

$$
X_{\mu}:\left\{\begin{array}{l}
\dot{x}=-y+k^{2} x^{2} y \\
\dot{y}=x-k^{2} x^{3}+\left(k^{2} x^{2}+k^{2} y^{2}-1\right) \\
\quad\left(-\frac{1}{2} \sum_{i=1}^{n} a_{2 i+1} y^{2 i+1}+\lambda y\left(1-k^{2} x^{2}\right)\right)
\end{array}\right.
$$

where $\mu=\left(k, \lambda, a_{3}, \ldots, a_{2 n+1}\right) \in \mathbb{R}^{n+2}, k \neq 0$

Lemma 1. For all $n \geq 1$ and for all $\mu=\left(k, \lambda, a_{3}, \ldots, a_{2 n+1}\right) \in \mathbb{R}^{n+2}$ the straight lines $L_{1}(x)=1-k x, L_{2}(x)=1+k x$ and the center $F(x, y)=k^{2} x^{2}+k^{2} y^{2}-1$ are invariant algebraic curve of (2.1). 
Proof. It is easy to verify that

$$
\dot{L_{1}}=L_{1_{x}} \dot{x}+L_{1_{y}} \dot{y}=k y L_{1} R(x, y),
$$

where the cofactor is $R(x, y)=k y(1+k x)$,

$$
\dot{L_{2}}=L_{2_{x}} \dot{x}+L_{2_{y}} \dot{y}=-k y L_{2} L(x, y),
$$

where the cofactor is $R(x, y)=-k y(1-k x)$, and for $F(x, y)=k^{2} x^{2}+k^{2} y^{2}-1$

$$
\dot{F}=F_{x} \dot{x}+F_{y} \dot{y}=F(x, y) R(x, y),
$$

where the cofactor is $R(x, y)=2 k^{2} y\left(-\frac{1}{2} \sum_{i=1}^{n} a_{2 i+1} y^{2 i+1}+\lambda y L_{1}(x) L_{2}(x)\right)$.

Then the straight lines $L_{1}(x), L_{2}(x)$ and the center $F(x, y)$ are invariant algebraic curve of (2.1).

Theorem 1. If $a_{2 j+1}=0, \forall j=1,2, \ldots, n-1$ and $a_{2 n+1} \neq 0$, system (2.1) at the origin has a weak focus of order $n$.

Proof. The linear part of $(2.1)$ at the singularity $(0,0)$ is

$$
D X_{\mu}(0,0)=\left(\begin{array}{ll}
0 & -1 \\
1 & -\lambda
\end{array}\right) \text {. }
$$

If $\lambda=0$, we have $\operatorname{div} X_{\mu}(0,0)=0$ and $\operatorname{det} D X_{\mu}(0,0)=1$, then the critical point $(0,0)$ is a weak focus. As $\operatorname{div} X_{\mu}(0,0)=-\lambda$, we have the Liapunov quantities $l_{0}=-\lambda$, and using Mathematica Software [1], we are able to compute the Liapunov quantities $l_{j}$, for $j \geq 1$. If $\lambda=0, l_{0}=0$ and $l_{1}=\frac{3 a_{3}}{16}$, if $a_{3}=0, l_{1}=0$ and $l_{2}=\frac{5 a_{5}}{32}$, if $a_{5}=0, l_{2}=0$ and $l_{3}=\frac{35 a_{7}}{256}$ and so on, then, If $\lambda=a_{3}=a_{5}=\ldots=$ $a_{2 n-1}=0$, and $a_{2 n+1} \neq 0$, we have $l_{0}=l_{1}=l_{3}=\ldots=l_{n-1}=0$, and for $n \geq 1$, $l_{n}=a_{2 n+1} \frac{(2 n+1) ! !}{2(2 n+2) ! !}$. Finally for all $j \geq n, l_{j}=a_{2 j+1} R_{j}(k)$ where $R_{j}(k)$ is a polynomial on $k$, then system (2.1) at the origin has a weak focus of order $n$.

Theorem 2. System (2.1) has a local center at the origin if and only if

$$
\lambda=a_{3}=a_{5}=\ldots=a_{2 n+1}=0 .
$$

Proof. From the linear part at the origin, it is clear that the condition $\lambda=0$ is necessary for a center. By Theorem 1, the Liapunov quantities are given by $l_{0}=-\lambda$ and for $n \geq 1, l_{n}=a_{2 n+1} \frac{(2 n+1) ! !}{2(2 n+2) ! !}$. As all calculated Liapunov quantities are zero, this shows that the conditions are necessary.

If $a_{2 j+1}=0, j=1,2,3, \ldots, n$, system (2.1) is given by

$$
\left\{\begin{array}{l}
\dot{x}=P(x, y)=-y+k^{2} x^{2} y \\
\dot{y}=Q(x, y)=x-k^{2} x^{3}
\end{array}\right.
$$


As the symmetries $P(-x, y)=P(x, y)$ and $Q(-x, y)=-Q(x, y)$ are satisfied, this proves the sufficiency that system (2.2) has a local center at the origin.

The solutions are on the circles centered at the origin and on the invariant straight lines $x=\frac{1}{k}$ and $x=-\frac{1}{k}$.

The small circles (local center), and large circles are solutions, and the large ones contain four nontrivial solutions plus four singularities. In between there is a circle containing two nontrivial solutions and two singularities.

In order to illustrate the result stated in Theorem 1, Figure 1 shows a numerical simulation of the system (2.1) with $k=1, a_{3}=0$ and $\lambda=0$, which corresponds to the case of center. The simulation was obtained using the Pplane7 Software with MATLAB [2].

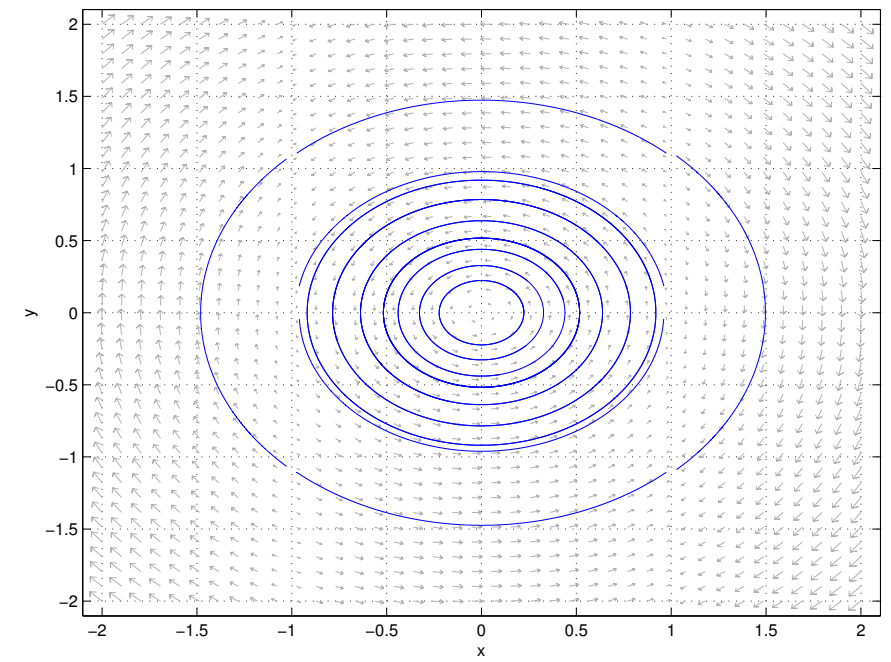

FIGURE 1. Local Center $(n=1)$.

Theorem 3. In the parameters space $\mathbb{R}^{n+2}$, there exists an open set $\mathcal{N} \neq \phi$, such that for all $\mu=\left(k, \lambda, a_{3}, \ldots, a_{2 n+1}\right) \in \mathcal{N}, k \neq 0$, system (2.1) has at least $n$ smallamplitude limit cycle bounded by the invariant conic.

Proof. If $\lambda=0$ and $a_{2 j-1}=0, \forall j=2,3, \ldots, n$ and $a_{2 n+1} \neq 0$, by Theorem 1 , system $(2.1)$ has at the singularity $(0,0)$ a repelling or attracting weak focus of order $n$. 
Let us consider the case $a_{2 n+1}>0$ and $n$ odd number, that is an attracting weak focus of order $n$. Taking $a_{2 n-1}<0$, so that $l_{n}>0$ and $l_{n-1}<0$, the origin of (2.1) is reversed and a hyperbolic repelling small amplitude limit cycle is created (Hopf $\mathrm{Bi}$ furcation), the limit cycle created persist under new small perturbation. Furthermore the singularity at the origin is an attracting weak focus of order $n-1$.

If we adjust the parameter $a_{2 n-3}$, so that $a_{2 n-3}>0$ small enough and $l_{n}>0$, $l_{n-1}<0$ and $l_{n-2}>0$, the stability at the origin is reversed and new hyperbolic attracting small amplitude limit cycle is created, that persists under new small perturbation. Furthermore the singularity at the origin is an repelling weak focus of order $n-2$. By following the same process until all Liapunov quantities are non-zero, such that in each step the stability of the singularity is inverted, $n$ hyperbolic small amplitude limit cycles are created and we obtain $n+1$ inequalities which define an a open set $\mathcal{N}$ in the parameter space, given by $\mathcal{N}=\left\{\left(k, \lambda, a_{3}, \ldots, a_{2 n+1}\right) \in \mathbb{R}^{n+2} \mid k \neq\right.$ $\left.0, l_{n}>0, l_{n-1}<0, l_{n-2}>0, \ldots, l_{2}<0, l_{1}>0, l_{0}<0\right\}$.

In order to illustrate the result stated in Theorem 3, Figure 2 shows a numerical simulation of the system (2.1) with $k=1, a_{3}=2$ and $\lambda=0.01$, which corresponds to the case of attracting limit cycle. The simulation was obtained using the Pplane7 Software with MATLAB [2].

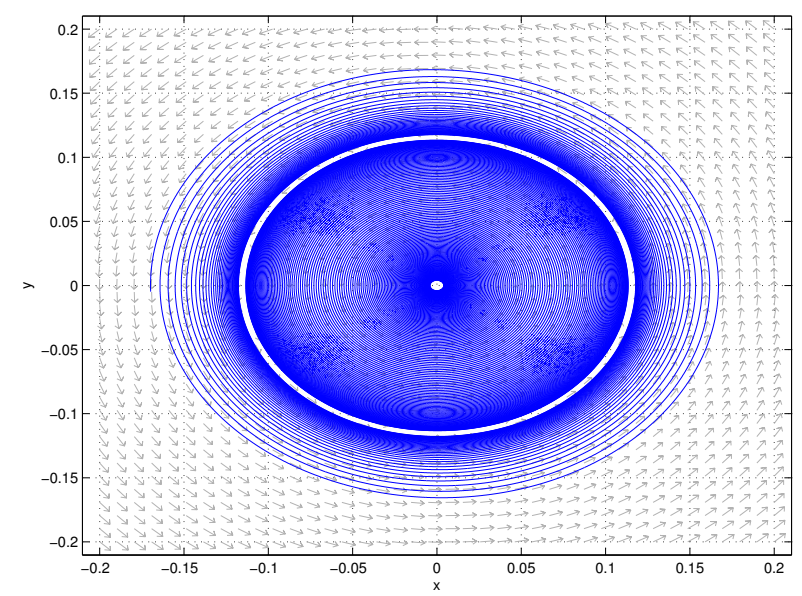

FIGURE 2. Repelling limit cycle $(n=1)$. 


\section{ACKNOWLEDGEMENT}

Z.H. Liu was supported by NNSF of China Grant No. 11671101 and Special Funds of Guangxi Distinguished Experts Construction Engineering.

\section{REFERENCES}

[1] Wolfram Research Mathematica: A System for Doing Mathematics by Computer, Champaign, IL, ser. Wolfram Research, Inc. Addison-Wesley, 1988.

[2] MATLAB: The Language of technical computing Using MATLAB (version 7.0). MatWorks, Natwick, MA, 2004.

[3] J. M. Hill, N. G. Lloyd, and J. M. Pearson, "Centers and limit cycles for an extended Kukles system," Electronic Journal of Differential Equations, vol. 2007, no. 119, pp. 1-23, 2007.

[4] I. S. Kukles, "Sur quelques cas de distinction entre un foyer et un centre," Dokl. Akad. Nauk. SSSR, vol. 42, no. 42, pp. 208-211, 1944.

Authors' addresses

\section{Zhenhai Liu}

Guangxi Key Laboratory of Universities Optimization Control and Engineering Calculation, and College of Sciences, Guangxi University for Nationalities, Nanning Guangxi 530006, P.R. China E-mail address: zhhliu@hotmail.com

\section{Iván Szántó}

Departamento de Matemática, Universidad Técnica Federico Santa María, Departamento de Matemática, Casilla 110-V, Valparaíso, Chile

E-mail address: ivan.szanto@usm.cl 